The submitted manuscript has been created by the University of Chicago as Operator of Argonne National Laboratory ("Argonne") under Contract No. W-31-109-ENG-38 with the U.S. Department of Energy. The U.S. Government retains for itself, and others acting on its behalf, a paid-up, nonexclusive, irrevocable worldwide license in said article to reproduce, prepare derivative works, distribute copies to the public, and perform publicly and display publicly, by or on behalf of the Government.

\title{
CTEQ5 Parton Distributions and Ongoing Studies
}

\section{S. Kuhlmann ${ }^{2}$}

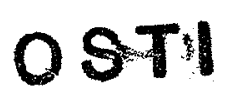

\author{
Argonne National Laboratory
}

The CTEQ5 parton distributions are described, with emphasis on the changes since CTEQ4. The most significant change is in the quark flavor dependence of the parton distributions. Ongoing studies of large-x parton distributions are discussed. Luminosity estimates are given for HERA in order to improve the present uncertainties of the quark distributions. A discussion of how to improve the gluon uncertainty in the future is presented.

\section{CTEQ5}

The gluon distribution is typically the most discussed of the proton's parton distributions, but the last two years have seen renewed interest in the flavor dependence of the quark distributions. This is due to new Tevatron asymmetry data from the Drell-Yan process, and also more precise ratio data from the NMC experiment. These new data were in disagreement with the older PDFs (which were based on lower statistics data from the same processes), hence a new analysis labeled CTEQ5 [1] was required.

The most dramatic change from earlier PDFs is in the ratio $\bar{d} / \bar{u}$, due to the fixed-target DrellYan asymmetry data from E866 (see ref. [1] for details). Figure 1 shows this ratio before and after this data was included. The new data prefers a turnover in the ratio at larger $x$, which could not have been predicted earlier. This is a superb example of how, in many cases, the uncertainty in parton distributions is dominated by the assumptions and parameterizations going into the fits, and not by experimental uncertainties.

The second most significant change since the CTEQ4 analysis is in the $d / u$ ratio. From $0.1<x<0.3$ the $W$ charge asymmetry data from CDF (see ref. [1] for details) has shown a clear need for a larger ratio, and the NMC deuteron/hydrogen ratio data shows this trend continues up to $x=0.65$, as shown in figure 2 . As discussed in the next section, possible nuclear effects in the deuteron would increase this ratio even further for $x>0.3$.

* Work supported by the U.S. Department of Energy, Division of High Energy Physics Contract $W-31-109-E N G-38$.

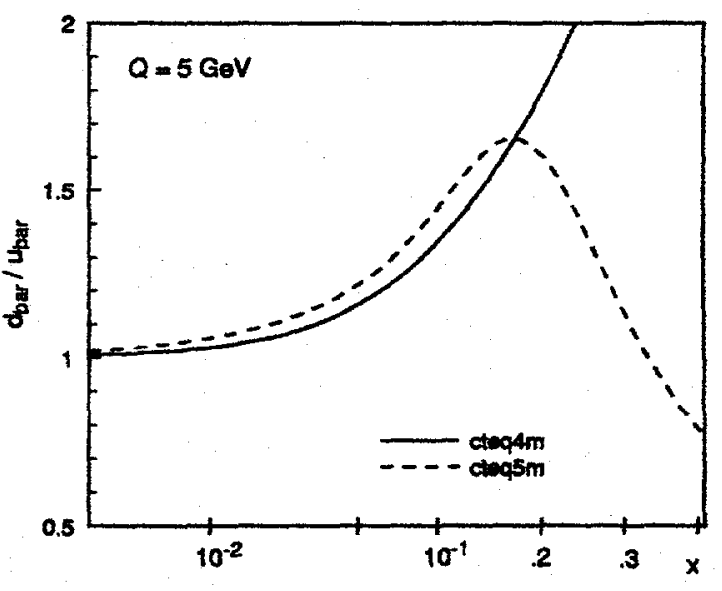

Figure 1. The ratio $\bar{d} / \bar{u}$ is shown for CTEQ4M and CTEQ5M.

\section{Large-X}

Four years have past since the CDF collaboration claimed an excess of high pt jets compared to "conventional" parton distribution sets. At that time the emphasis was on the uncertainty in the large-x gluon distribution. This uncertainty has not decreased in the last four years, in fact it has perhaps increased with the latest analyses from CTEQ and MRS. This is demonstrated in fig. 3. This plot shows ratios of gluon distributions, at $Q=5 \mathrm{GeV}$ on top and at $Q=100 \mathrm{GeV}$ on bottom. The denominator is always the CTEQ4M parton distributions. The solid lines are the result of a 


\section{DISCLAIMER}

This report was prepared as an account of work sponsored by an agency of the United States Government. Neither the United States Government nor any agency thereof, nor any of their employees, make any warranty, express or implied, or assumes any legal liability or responsibility for the accuracy, completeness, or usefulness of any information, apparatus, product, or process disclosed, or represents that its use would not infringe privately owned rights. Reference herein to any specific commercial product, process, or service by trade name, trademark, manufacturer, or otherwise does not necessarily constitute or imply its endorsement, recommendation, or favoring by the United States Government or any agency thereof. The views and opinions of authors expressed herein do not necessarily state or reflect those of the United States Government or any agency thereof. 


\section{DISCLAIMER}

Portions of this document may be illegible in electronic image products. Images are produced from the best available original document. 


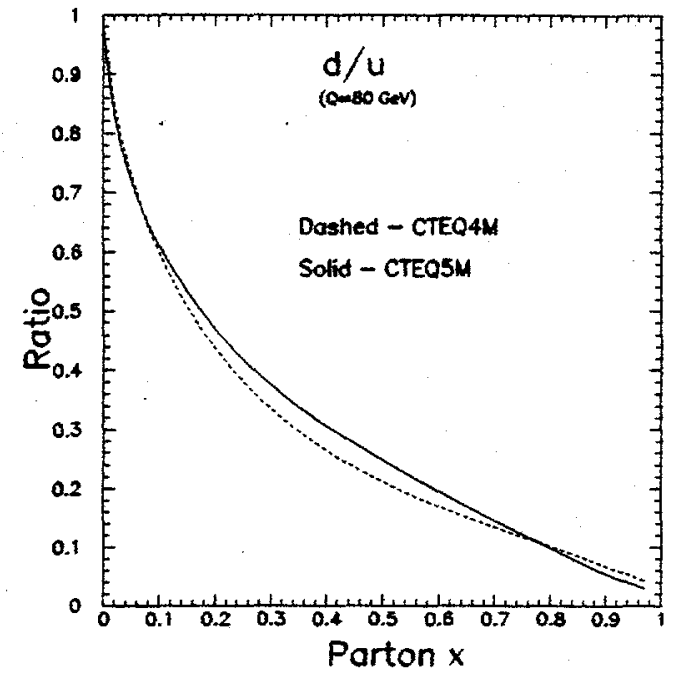

Figure 2. The ratio $d / u$ is shown for CTEQ4M and CTEQ5M.

CTEQ gluon parameter scan, and are meant to represent an approximate uncertainty band. The other lines are the latest fits from CTEQ and MRS. CTEQ5M is not shown but is very close to CTEQ4M. One sees that all the ghons agree very well below $x<0.1$ and at reasonable $Q$ values such as $100 \mathrm{GeV}$, while above $x>0.1$ more work is clearly needed to reduce the uncertainties.

In the last two years the focus has changed, with the possible uncertainty of large-x quark distributions being reevaluated. The jet data itself is mostly quark scattering, so a $5 \%$ change in quark distributions can mimic a 50three possible ways to modify the quark distributions: 1) modify them dramatically near $x=1$ and then this change will evolve downward to lower $x$ as $Q$ increases, 2) higher twist effects, and 3) the $d / u$ ratio at large-x. In this brief report we will only discuss the $d / u$ ratio.

An analysis of SLAC experiment E139 led to the conclusion that the infamous "EMC Effect" was present in deuterium as well. It would not be too surprising if this were the case, but this con-

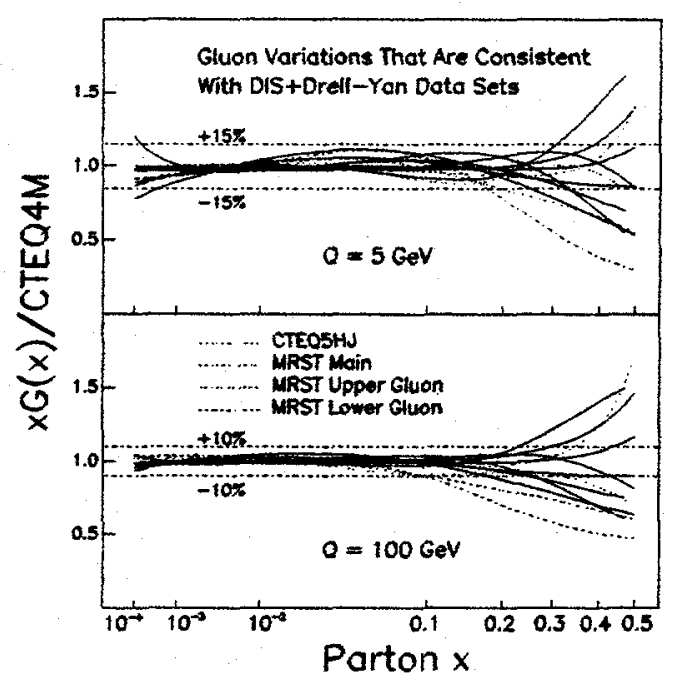

Figure 3. The results of a gluon parameter scan are shown, as ratios with respect to CTEQ4M gluons (solid lines). These represent an uncertainty band for the gluon at these $x$ and $Q$ values. Also shown are the latest gluon distributions from MRST. The updated CTEQ5M gluons are very close to CTEQ4M and are not shown, while the special increased gluon fit called CTEQ5HJ is shown.

clusion depends on an unproven nuclear physics model and numerous fitted parameters. Therefore we consider this yet another uncertainty at large- $x$ to be evaluated. To do this we refit the parton distributions with the full corrections derived from E139. We find we get equally good fits with or without these corrections, but the d/u ratio changes dramatically at large-x. This is shown in fig. 4. The best way to settle this issue is with charged current data at HERA. However, it will take a considerable data set coming from the upgraded machine to do this. A group from ZEUS [3] estimates $250 \mathrm{pb}^{-1}$ per detector is needed to get a $2 \sigma$ separation between these curves. 


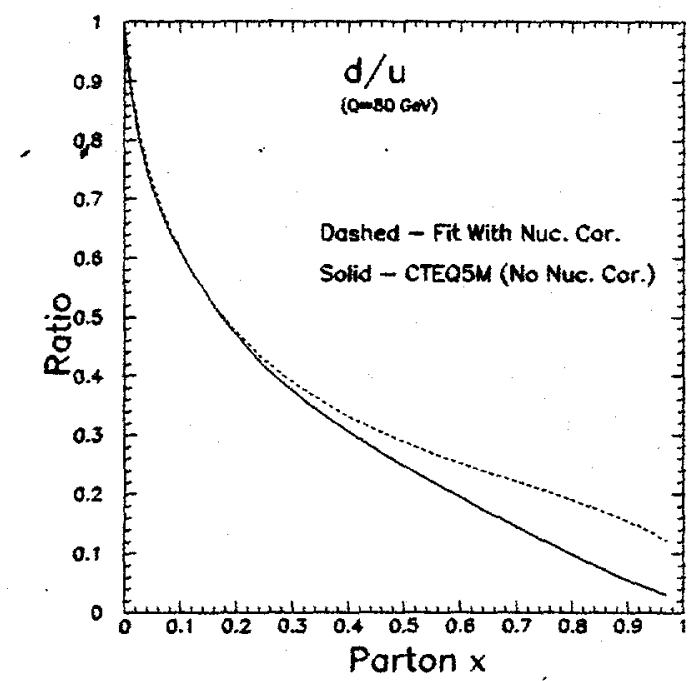

Figure 4. The $d / u$ ratio is shown for CTEQ5M and for a special fit using deuteron nuclear corrections.

\section{Future Proopects}

The uncertainties in large-x quarks and gluons need to be reduced or else physics in this region will be compromised for the Tevatron and LHC. Jet data from the Tevatron, especially the expected triply differential dijet measurements, will provide strong contraints on large- $x$ partons but will not be able to distinguish changes in quarks or gluons. Therefore it is crucial for HERA to collect enough data to settle all the issues for the quarks, after which the gluons can be determined from jets or direct photons.

\section{REFERENCES}

1. H.L. Lai et al., hep-ph/9903282

2. A.D. Martin et al., Eur. Phys. J. C4 (1998) 463

3. M. Kuze, K. Nagano, A. van Sighem (private communication) 